\title{
Low-x Physics at a Future Electron-Ion Collider (EIC) Facility
}

\author{
Bernd Surrow \\ Massachusetts Institute of Technology, Department of Physics, Laboratory for Nuclear Science \\ 77 Massachusetts Avenue, Cambridge, MA 02139
}

The proposed polarized electron-ion collider (EIC) will allow for precision exploration of various novel aspects of QCD including low-x phenomena, the spin structure of the proton, and gluon saturation in heavy nuclei. As this project gains momentum, it is increasingly important for the QCD community to understand quantitatively the kinematic reach and expected sensitivities for various measurements. We briefly summarize key accelerator design parameters and then focus on expected measurement sensitivities, thus exposing how the EIC will allow an extension of the successful HERA program into exciting new regimes.

\section{Introduction}

QCD is a spectacularly successful theory, yet it remains an active field of research in particle physics. While it has withstood over several decades of tests, we have yet to understand fully the mechanisms by which complex and rich phenomena emerge from a theory based solely on symmetry and local gauge invariance [2]. This quest is being pursued on many fronts: hadron structure, hadron spectroscopy, high temperature phenomena and high parton density systems. There is little doubt that our current understanding of QCD, in particular the role of gluons in QCD dynamics, has been greatly advanced by using electron-proton collisions at the HERA collider, which was the first facility to probe with high luminosity deep into the regime where gluons play a dominant role in hadron structure. In the following, we discuss how a high luminosity Electron-Ion Collider (EIC) will enable similar advances in the study of strong color fields, in particular many body gluon states probed at the saturation scale. The crucial element in these studies will be the use of heavy nuclei that, when probed at small Bjorken $x$, amplify such novel gluon states.

\section{Opportunities in low-x physics at a future EIC facility}

From deep inelastic scattering (DIS) we know that gluons carry half of the momentum of the proton, and from HERA we know that gluons dominate for $x<0.01$ [3]. However, when probed at low- $x$, it is predicted that the gluon distribution within a proton will saturate. There are many quantitative arguments for this, but it is rather intuitive that at sufficiently high gluon density $2 \rightarrow 1$ gluon fusion (non-linear dynamics) will begin to dominate over $1 \rightarrow 2$ gluon splitting (linear dynamics). This transition occurs at a scale commonly referred to as the saturation scale $\left(Q_{s}\right)$. Geometrical considerations [4] show that, in nuclei, $Q_{s} \propto A^{1 / 3} x^{-\delta}$, where $\delta \sim 1 / 3[5]$. Thus, heavy nuclei can be used to amplify the scale at which gluon saturation sets in. Figure 1 shows the saturation scale in the $Q^{2}$ versus $x$ plane for ions ranging from protons to $\mathrm{Au}[3]$. Additionally, the colored diagonal lines show the kinematic limits of the EIC for various beam energies. The shaded band illustrates the accessible saturation region for $20 \mathrm{GeV}$ electrons colliding with $100 \mathrm{GeV}$ Au nuclei. The saturation 


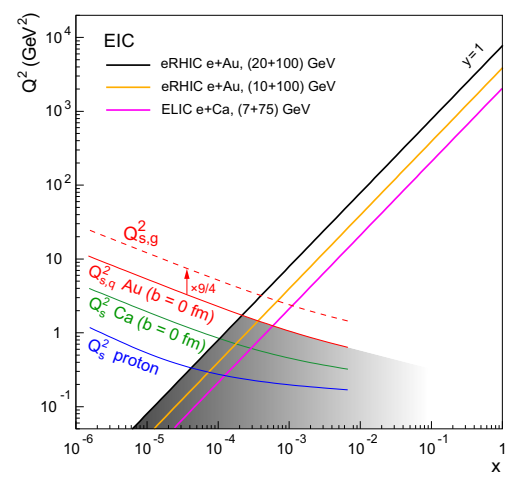

Figure 1: The kinematic plane of DIS variables $x$ and $Q^{2}$. The straight diagonal lines represent the kinematic boundaries of the EIC under various beam configurations. Regions below the lines are accessible. The curved/dashed lines indicate the saturation scale for various ions. Color online.

scale for gluons in $\mathrm{Au}$ can be accessed at a factor of 10 larger $x$ and $Q^{2}$ than $Q_{s}$ for gluons in a proton. This has two significant impacts. First, by substituting heavy ions for protons, one can access the saturation regime at lower beam energies. Second, $Q_{s, g}^{2}(A u)>Q^{2}>\Lambda_{Q C D}^{2}$, thereby enabling use of perturbative methods for calculation. The saturation regime can be reached with reasonably modest beam energies, and it can be explored with the use of perturbative calculations.

In DIS the differential cross section $d^{2} \sigma / d x d Q^{2}$ can be decomposed in terms of two structure functions $\left(F_{2}\left(x, Q^{2}\right)\right.$ and $\left.F_{L}\left(x, Q^{2}\right)\right)$, where $F_{2}$ is directly sensitive to (anti)quark distributions, and $F_{L}$ is directly proportional to the gluon momentum distribution. These structure functions provide a direct means to quantitatively study saturation phenomena and we discuss them in more detail below. In the following we show the measurement prospects for various EIC configurations with a maximum center-of-mass energy of $\sqrt{s}=14 \sim 140$ $\mathrm{GeV} / \mathrm{n}$ and a maximum luminosity $L=10^{33} \sim 10^{35} \mathrm{~cm}^{-2} \mathrm{~s}^{-1}$, a factor of 100 higher than HERA. As the world's first electron heavy ion collider, the EIC would enable the high precision exploration of $F_{2}$ and $F_{L}$ of heavy Nuclei in the saturation regime, truly terra incognita. As we will show, the luminosity, energy and collider kinematics will be used to differentiate competing models of low- $x$ QCD phenomena.

One of the first measurements at the EIC will be $F_{2}\left(x, Q^{2}\right)$ for both heavy $(\mathrm{Au})$ and light (d) ions. The ratio, shown versus $Q^{2}$ for four $x$ bins, is shown in Figure 2 [3]. The points represent the anticipated statistical precision achievable with an integrated luminosity of 4/A $\mathrm{fb}^{-1}$. The colored lines are predictions from competing models [3]. $F_{2}$ is directly sensitive to quark distributions, and is sensitive to gluons via scaling violations. nDS, EKS and FGS are pQCD models with differing treatment of parton shadowing, and they are compared to predictions from the Color Glass Condensate (CGC) model. Within the expected precision of the measurements, differentiation between the different models is clearly possible in the region $10^{-4}<x<10^{-2}$.

With the ability to accelerate both light and heavy ions over a wide range of energies, the EIC will be able to make significant contributions to the understanding of the gluon distribution in the proton. At low $x, F_{L}\left(x, Q^{2}\right) \propto \alpha_{s} x G\left(x, Q^{2}\right)$, where $G\left(x, Q^{2}\right)$ is the 


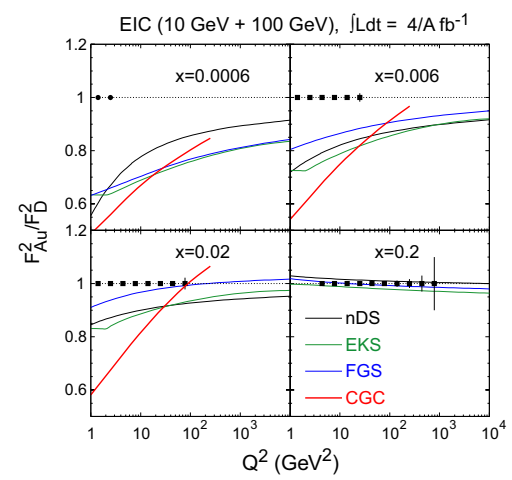

Figure 2: The ratio of $F_{2}\left(Q^{2}\right)$ in Au to that in d nuclei, for four bins in $x$. The symbols represent the anticipated statistical precision achievable. The curves represent models with differing treatment of low- $x$ phenomena. Color online.

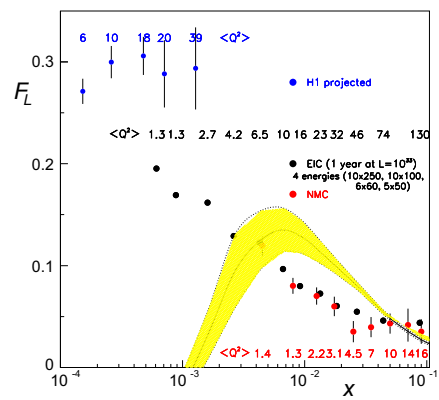

Figure 3: $F_{L}(x)$ for protons from fixed target (red), projected H1 (blue) and projected EIC (black). Color online.

gluon distribution. Extraction of $F_{L}$ requires running at multiple beam energies, a task that highlights the flexibility of the EIC. Figure 3 shows $F_{L}(x)$ for a proton. The red points are from existing NMC fixed target data, and the blue $\mathrm{H} 1$ points show the expected precision (statistical and systematic uncertainties) achievable from the recent HERA energy scan [3]. The black points show the achievable precision from one year of running the EIC at four different energies (statistical uncertainties only) [3]. The EIC measurements will clearly compliment the HERA results, as well as bridge the region between HERA and fixed target results, contributing to a direct measurement of the gluon distribution for $10^{-4}<x<10^{-1}$.

Finally, Figure 4 shows the ratio of gluon distributions in $\mathrm{Pb}$ to that in $\mathrm{d}\left(R_{g}^{P b}=\right.$ $\left.F_{L}^{P b} / F_{L}^{d} \sim G^{P b} / G^{d}\right)$ [3]. Current data on the gluon distribution at low- $x$ in heavy nuclei is sparse. In turn, constraints on theoretical models are weak, as shown by the vast range of different theoretical predictions. The gluon distribution is critical for accurate calculations of cross sections at both RHIC and the LHC. With its high luminosity, the EIC can make significant contributions to the understanding of the gluon distribution in heavy nuclei over 


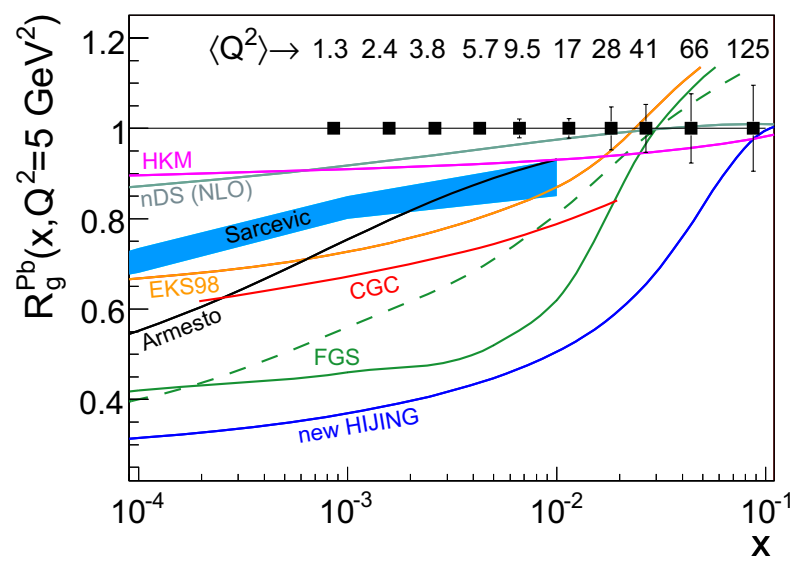

Figure 4: The ratio of $F_{L}$ in $\mathrm{Pb}$ to that in d. The black symbols represent anticipated statistical uncertainty from $10 / \mathrm{A} \mathrm{fb}^{-1}$ of EIC data, compared to the large range of model predictions over this currently unexplored kinematic range. Color online.

the relevant $x$ range.

\section{Summary}

In conclusion, as the world's first high energy electron (heavy) ion collider, and with a luminosity approximately one hundred times that of HERA, the EIC will allow precision exploration of strong color fields. The use of heavy nuclei will amplify the scale at which saturation phenomena are predicted, placing it well within the accessibility of the EIC. There are many topics we have neglected to discuss, in particular diffraction, spin decomposition of the proton, and the study of partonic energy loss in cold nuclear matter. The physics program of the EIC is rich, diverse, and well targeted toward a unified understanding of strongly interacting matter. The project is gaining momentum on an international scale and will provide a continuation of the successful HERA program into exciting new regimes of QCD.

\section{References}

[1] Slides:

http://indico. cern. ch/contributionDisplay py? contribId=263\&sessionId=10\&conf Id=9499

[2] F. Wilczek. Nucl. Phys. Proc. Suppl., 119:3, 2003.

[3] EIC Collaboration. A high luminosity, high energy electron-ion collider, white paper prepared for the nuclear science advisory committee as input to the long-range planning 2007.

[4] K. Golec-Biernat and M. Wustoff. Phys. Rev., D59:014017, 1999.

[5] C. A. Salgado N. Armesto and U. A. Wiedemann. Phys. Rev., 94:0220002, 2005. 